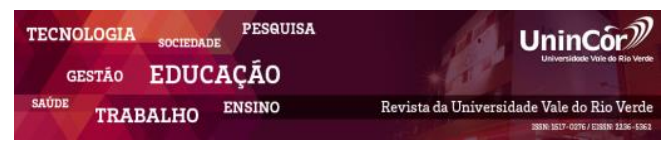

Revista da Universidade Vale do Rio Verde ISSN: 1517-0276 / EISSN: 2236-5362 v. $17 \mid$ n. $1 \mid$ Ano 2019

Rejane Mendes Costa Borges Faculdades de Saúde Ibituruna, FASI nandesenfg@gmail.com

Maria Cecília Andrade Silva Faculdade de Saúde Ibituruna, FASI simone.souto@funorte.edu.br

Simone Valéria Dias Souto Faculdade Verde Norte, FAVENORTE simonevds03@yahoo.com.br

Ernandes Gonçalves Dias Faculdade Verde Norte, FAVENORTE ernandesgdias@yahoo.com.br

\section{PERFIL ANTROPOMÉTRICO E HÁBITOS ALIMENTARES DE \\ BOMBEIROS DE UM BATALHÃO EM MINAS GERAIS, BRASIL}

\section{RESUMO}

A avaliação antropométrica e dos hábitos alimentares possibilita obtenção de dados que auxiliam na prevenção de condições crônicas não transmissíveis, sobretudo entre bombeiros que devem estar saudáveis para exercer suas funções, que exigem preparo físico. Objetivou-se avaliar o perfil antropométrico e os hábitos alimentares de bombeiros militares de Montes ClarosMG. Trata-se de um estudo descritivo e quantitativo realizado com 54 bombeiros a partir da mensuração de dados antropométricos e aplicação de questionários de "Anamnese" e de "Frequência Alimentar" em setembro de 2017 e analisados estatisticamente com auxílio Programa Statiscal Package for the Social Science. Observou-se que $87,03 \%$ dos participantes eram do sexo masculino, 59,25\% exerciam atividade administrativa, $98,15 \%$ eram praticantes de atividade física, $42,59 \%$ apresentaram sobrepeso e a relação circunferência cintura/quadril da maioria se enquadrou na classificação de moderado a alto risco para homens e mulheres, respectivamente, para doenças cardiovasculares. O percentual de gordura, em ambos os sexos, foi classificado como alto para a maioria. Em relação aos hábitos alimentares observou-se consumo de alimentos calóricos, poucos alimentos ricos em fibras e pouca variação no consumo de hortaliças e frutas. Conclui-se ser importante a programação de atividades de intervenções preventivas, com enfoque nos hábitos alimentares para que os problemas relacionados a dieta inadequada e ganho de peso sejam controlados a fim de evitar que os bombeiros sejam impedidos de atuarem em função do agravamento de fatores de risco cardiovasculares, obesidade e surgimento de condições crônicas de saúde não transmissíveis associadas ao estilo de vida e ao padrão antropométrico.

Palavras-chave: Antropometria. Comportamento alimentar. Avaliação Nutricional. Bombeiros. Militares.

\section{ANTHROPOMETRIC PROFILE AND FOOD HABITS OF FIREFIGHTERS OF A BATTALION IN MINAS GERAIS, BRAZIL}

\begin{abstract}
Anthropometric evaluation and eating habits make it possible to obtain data that help prevent chronic non-communicable conditions, especially among firefighters who must be healthy to perform their functions, which require physical training. The objective was to evaluate the anthropometric profile and feeding habits of the Montes Claros-MG military firefighters. This is a
\end{abstract}


descriptive and quantitative study carried out with 54 firefighters from the measurement of anthropometric data and the application of "Anamnesis" and "Food Frequency" questionnaires in September 2017 and analyzed statistically with assistance Statiscal Package Program for the Social Science. It was observed that $87.03 \%$ of the participants were males, $59.25 \%$ were administrative, $98.15 \%$ were physical activity practitioners, $42.59 \%$ were overweight and the waist-to-hip circumference ratio of the majority was framed in the classification of moderate to high risk for men and women, respectively, for cardiovascular diseases. The percentage of fat, in both sexes, was classified as high for the majority. In relation to eating habits, caloric food consumption, few fiber-rich foods and little variation in the consumption of vegetables and fruits were observed. It is concluded that it is important to program preventive intervention activities focusing on eating habits so that problems related to inadequate diet and weight gain are controlled in order to prevent firemen from being able to act due to the aggravation of risk factors cardiovascular diseases, obesity and the appearance of chronic non-transmissible health conditions associated with lifestyle and anthropometric standard.

Keywords: Anthropometry. Feeding Behavior. Nutrition Assessment. Firefighters. Military Personnel.

Recebido em: 18/01/2019 - Aprovado em: 20/03/2019 - Disponibilizado em: 15/07/2019

\section{INTRODUÇÃO}

Na maioria das profissões, um bom desempenho esta relacionado com o estado físico e nutricional do trabalhador, visto que a fonte de energia para que exerça corretamente suas atividades é o alimento, assim, os hábitos alimentares de um indivíduo repercutem em seu estado de saúde (CONFORTIN; SOEIRO, 2014). Nesse sentido, Santos Neto, Cavalcante Neto e Pinto (2014) afirmam que uma vida saudável é essencial dedicar-se às atividades físicas e momentos de lazer que favorecem redução do estresse e prevenção às Doenças Crônicas Não Transmissíveis (DCNT).

Atualmente tem sido considerado normal ter horas excessivas de trabalho, mais de um emprego, acumular tarefas e levar serviço para casa, entretanto em algumas profissões, os profissionais necessitam estar bem condicionados para cumprir sua função, como é o caso de militares que se arriscam para salvar a vida do próximo e a si mesmo (KINDINGER, 2015). Nesse sentido, em relação ao bombeiro militar, espera-se que tenha excelente preparação física para realizar as tarefas inerentes ao cargo.

Segundo Jesus et al. (2015) a atividade física é um fator de proteção contra doenças, pois beneficia o controle dos níveis pressóricos, reduz o estresse, a fadiga, além de atuar no controle do estado nutricional. Contudo, a rotina diária da população tem intensificado o surgimento de condições crônicas de saúde, que além da falta de prática de atividade física, estão também associadas à falta de lazer, alimentação inadequada e a dificuldades socioeconômicas.

Na opinião de Silva, Assis e Silva (2014) o sedentarismo associado a uma má alimentação e o atual ritmo de vida da população, são fatores de 
riscos para obesidade, doenças e desempenho profissional. A adequação do perfil nutricional de militares é necessária para o desenvolvimento satisfatório em suas funções e resultam em melhoria da qualidade de vida.

Nesse sentido, Confortin e Soeiro (2014) reforçam que um bombeiro exerce diversas funções dentre elas burocráticas, em escritório, e ações extremas de combate, sendo assim o preparo físico é essencial para garantir a execução de seus trabalhos, dessa forma a composição corporal é um indicador de saúde, pois o excesso de gordura corporal interfere na capacidade física.

De acordo Damasceno et al. (2016) a antropometria é o método mais utilizado na avaliação de pessoas já que é um método não invasivo, de fácil entendimento e baixo custo, além de ser citado como excelente ferramenta para verificar composição corporal e útil para diagnosticar problemas de saúde cardiovascular. Ainda afirma que a avaliação antropométrica é de grande valia no diagnóstico de riscos nutricionais, uma vez que a porcentagem de gordura está relacionada a problemas metabólicos do organismo e elevação dos riscos de surgimento das DCNT que para além da hipertensão arterial, compreende as úlceras gastroduodenais, obesidade, câncer e outras, que podem de alguma forma ser relacionadas às atividades laborais dos militares.

Dessa maneira, face às mudanças na rotina das pessoas pode-se observar que em todos os seguimentos profissionais, as mudanças comportamentais, têm promovido alterações nas características corporais, como aumento da gordura abdominal. A avaliação dos hábitos alimentares e antropométrica é importante para entender e re- verter às condições de alteração do estado de saúde dos bombeiros militares, pois é importante que esses profissionais estejam preparados física, nutricional e psicologicamente para que a sociedade tenha um serviço prestado com excelência. Dessa forma, o objetivo desse estudo foi avaliar o perfil antropométrico e os hábitos alimentares de bombeiros militares de Montes Claros, Minas Gerais.

\section{MÉTODOS}

Estudo de caráter descritivo e natureza quantitativa, realizado com 54 bombeiros militares do $7^{\circ}$ Batalhão do Corpo de Bombeiros Militar de Minas Gerais sediado na cidade de Montes Claros, pertencentes ao quadro de militares da ativa.

Foram incluídos no estudo os militares presentes no período da coleta de dados e que consentiram sua participação na pesquisa como determina a Resolução 466/2012.

Para coleta dos dados foi aplicado o questionário de anamnese e frequência alimentar, em um local reservado e em horário previamente agendado. A coleta de dados foi realizada no mês de setembro de 2017, pelos próprios pesquisadores em espaço disponibilizado pela instituição.

Para coleta dos dados foi utilizada balança digital da marca Tech Line® com capacidade de até $180 \mathrm{~kg}$ e graduação de $100 \mathrm{~g}$, estadiômetro e adipômetro da marca Cardiomed, fita métrica inelástica da marca Avanutri, todos os instrumentos aprovados pelo Instituto Nacional de Metrologia (Inmetro). 
Para a avaliação antropométrica foi utilizado o protocolo de IMC (WHO1999). Na avaliação da composição corporal foi seguido o Protocolo de Durnin e Wormersley (1974), para obtenção da somatória das pregas cutâneas $(\Sigma 4$ $\mathrm{PC}=\mathrm{PCT}+\mathrm{PCB}+\mathrm{PCSE}+\mathrm{PCSI}) \cdot \mathrm{E} \mathrm{a}$ classificação da porcentagem de gordura seguiu os parâmetros de Pollock e Wilmore (1993), com diferenciação para homens e mulheres. A Circunferência da Cintura (CC) foi mensurada na região da cintura acima do umbigo e a circunferência do quadril foi obtida com o militar na maior proeminência do glúteo, obtendo-se a Relação Cintura Quadril (RCQ). Por meio de adipômetro foram obtidas as pregas subescapular, tricipital, biciptal e suprailíaca.

Também foi utilizado um questionário de Anamnese do Instituto Federal de Educação, Ciências e Tecnologia do Rio grande do Sul, adaptado por estes pesquisadores, com nove (09) questões contendo dados sociodemográficos e de saúde e outro Questionário de Frequência de Consumo Alimentar com nove (09) itens, elaborado por estes pesquisadores com base nos grupos alimentares: cereais, hortaliças, frutas, leites e derivados, leguminosas, carnes e ovos, óleos e gorduras, açúcares e outros que não se enquadram nos grupos.

Todos os dados coletados foram reunidos e armazenados em uma planilha no Software Excel 2013 depois foi utilizado a versão 20.0 do programa Statiscal Package for the Social Science (SPSS) para análise.

$\mathrm{O}$ projeto de pesquisa desse estudo foi submetido ao Comitê de Ética e Pesquisa da
Associação Educativa do Brasil (SOEBRAS) e aprovado Parecer Consubstanciado no 2.151.113.

\section{RESULTADOS}

Na tabela 1 estão apresentados os dados sóciodemográficos e as características de saúde e laborais dos bombeiros.

Observou-se que a maioria dos participantes era do sexo masculino (47; $87,03 \%)$, trabalhavam em atividades administrativas $(32 ; 59,25 \%)$ e na atividade operacional $(22 ; 40,75 \%)$. Com relação à prática de atividades física $53(98,15 \%)$ afirmaram ser praticantes, enquanto $01(1,85 \%)$ se caracterizou como não praticante. Entre os praticantes de atividade física $21(38,90 \%)$ praticante entre 1 e 5 anos, 09 (16,66\%) entre 06 e 10 anos, 12 $(22,22 \%)$ há mais de 10 anos ao passo que 11 $(20,37 \%)$ não souberam determinar a quanto tempo são praticantes de atividade física.

Em relação ao tempo de sono, 34 $(62,97 \%)$ participantes dormem menos de 8 horas/noite, enquanto $19(35,18 \%)$ responderam dormir 8 horas/noite e $01(1,85 \%)$ dorme menos de 08 hora/noite. Sobre tabagismo $53(98,15 \%)$ responderam não fumar, porém 41 (75,93\%) afirmaram ser etilistas.

Quanto à presença de condições crônicas não transmissíveis, 03 (5,55\%) são portadores de hipertensão, 03 (5,55\%) de colesterol elevado, 03 $(5,55 \%)$ de trigliceres elevado, $03(5,55 \%)$ tem mais de uma das condições citadas anteriormente associadas, contudo $42(77,80 \%)$ responderam não ser portador de nenhuma condição crônica de saúde. 
Tabela 1 - Dados sociodemográficos e características de saúde e laborais de Bombeiros sediados em Montes Claros, Minas Gerais, 2017.

\begin{tabular}{|c|c|c|c|}
\hline \multicolumn{2}{|r|}{ Variáveis } & \multirow{2}{*}{$\frac{\mathbf{N}(\mathbf{5 4})}{07}$} & \multirow{2}{*}{$\frac{\mathbf{F}(\%)}{12,97}$} \\
\hline \multirow{2}{*}{ Sexo } & Feminino & & \\
\hline & Masculino & 47 & 87,03 \\
\hline Tipo de & Administrativa & 32 & 59,25 \\
\hline Atividade & Operacional & 22 & 40,75 \\
\hline \multirow{2}{*}{$\begin{array}{c}\text { Prática de } \\
\text { Atividade Física }\end{array}$} & Sim & 53 & 98,15 \\
\hline & Não & 01 & 1,85 \\
\hline \multirow{5}{*}{$\begin{array}{c}\text { Tempo de prática } \\
\text { de atividade } \\
\text { física }\end{array}$} & 1 a 5 anos & 21 & 38,90 \\
\hline & 6 a 10 anos & 09 & 16,66 \\
\hline & Acima de 10 anos & 12 & 22,22 \\
\hline & Não sabe & 11 & 20,37 \\
\hline & Não pratica & 01 & 1,85 \\
\hline \multirow{3}{*}{$\begin{array}{c}\text { Sono } \\
\text { (Horas/noite) }\end{array}$} & $<8$ horas & 34 & 62,97 \\
\hline & 8 horas & 19 & 35,18 \\
\hline & $>8$ Horas & 01 & 1,85 \\
\hline \multirow{2}{*}{ Tabagismo } & Sim & 01 & 1,85 \\
\hline & Não & 53 & 98,15 \\
\hline \multirow{2}{*}{ Etilismo } & Sim & 41 & 75,93 \\
\hline & Não & 13 & 24,07 \\
\hline \multirow{5}{*}{$\begin{array}{l}\text { Condições } \\
\text { Crônicas }\end{array}$} & Hipertensão & 03 & 5,55 \\
\hline & Colesterol elevado & 03 & 5,55 \\
\hline & Trigliceres elevado & 03 & 5,55 \\
\hline & Não possui & 42 & 77,80 \\
\hline & Associação de condições citadas acima & 03 & 5,55 \\
\hline
\end{tabular}

Fonte: Elaborado pelos autores, 2017.

A Tabela 2 apresenta os dados do perfil antropométrico dos bombeiros avaliados.

Observou-se que o quantitativo de militares com peso adequado (46,30\%) e sobrepeso $(42,59)$ é similar, no entanto entre o conjunto analisado $53,7 \%$ apresentaram alteração de IMC. A relação circunferência da cintura das mulheres bombeiros foi classificada como sem risco $(100 \%)$, com essa classificação aparece 38 $(80,85 \%)$ homens, enquanto que 06 e 03 homens apresentam risco elevado e muito elevado para doenças cardiovasculares, respectivamente.

A relação circunferência cintura/quadril entre as mulheres apresentou uma maior porcentagem de alto risco para comorbidades associadas à obesidade $(57,14 \%)$. Entre os homens o índice variou entre baixo, moderado e alto risco, sendo o moderado o de maior frequência $(51,06 \%)$.

Tabela 2- Perfil Antropométrico e Nutricional dos Bombeiros militar de Montes Claros, MG, 2017.

\begin{tabular}{cccc}
\hline & Variáveis & $\mathbf{N}(\mathbf{0 7}$ mulheres e 47 homens $)$ & $\mathbf{F}(\boldsymbol{\%})$ \\
\hline \multirow{3}{*}{ IMC } & Eutrófico & 25 & 46,30 \\
& Sobrepeso & 23 & 42,59 \\
& Obesidade I & 05 & 9,26 \\
& Obesidade II & 01 & 1,85
\end{tabular}




\begin{tabular}{|c|c|c|c|}
\hline & \multicolumn{3}{|c|}{ Mulheres } \\
\hline & Bom & 01 & 14,28 \\
\hline & Moderadamente & 01 & 14,28 \\
\hline \multirow{5}{*}{$\%$ Gordura } & alto & & \\
\hline & Muito alto & 03 & 42,86 \\
\hline & Alto & 02 & 28,58 \\
\hline & \multicolumn{3}{|c|}{ Homens } \\
\hline & Muito baixo & 02 & 4,26 \\
\hline \multirow{6}{*}{$\%$ Gordura } & Bom & 07 & 14,90 \\
\hline & Muito bom & 04 & 8,51 \\
\hline & Adequado & 01 & 2,12 \\
\hline & $\begin{array}{l}\text { Moderadamente } \\
\text { alto }\end{array}$ & 08 & 17,02 \\
\hline & Alto & 15 & 31,91 \\
\hline & Muito alto & 10 & 21,28 \\
\hline \multirow{6}{*}{$\begin{array}{l}\text { Circunferênci } \\
\text { a da Cintura }\end{array}$} & \multicolumn{3}{|c|}{ Mulheres } \\
\hline & Sem risco & 07 & 100 \\
\hline & \multicolumn{3}{|c|}{ Homens } \\
\hline & Sem risco & 38 & 80,85 \\
\hline & Risco elevado & 06 & 12,77 \\
\hline & Risco muito elevado & 03 & 6,38 \\
\hline \multirow{7}{*}{ RCQ } & \multicolumn{3}{|c|}{ Mulheres } \\
\hline & Baixo & 03 & 42,86 \\
\hline & Alto & 04 & 57,14 \\
\hline & \multicolumn{3}{|c|}{ Homens } \\
\hline & Baixo & 19 & 40,43 \\
\hline & Moderado & 24 & 51,06 \\
\hline & Alto & 04 & 8,51 \\
\hline
\end{tabular}

Fonte: Elaborado pelos autores, 2017.

Os alimentos mais frequentes na alimentação dos bombeiros estão categorizados e apresentados na Tabela 3. Observou-se consumo de alimentos calóricos, poucos alimentos ricos em fibras e pouca variação no consumo de hortaliças e frutas.

O leite integral e o óleo vegetal são os alimentos menos consumidos e o café o alimento de maior consumo pelos bombeiros.

O grupo das hortaliças e de outros alimentos, onde aparecem alimentos como café e salgados, parece concentrar uma maioria de adeptos bombeiros na consumação de alimentos.

Tabela 3 - Alimentos mais consumidos pelos bombeiros militar de Montes Claros-MG, 2017.

\begin{tabular}{clcl}
\hline \multicolumn{2}{c}{ Variáveis } & N (=54) & Frequência \\
\hline \multirow{6}{*}{ Cereais } & Arroz & 25 & 2 ou mais vezes ao dia \\
& Macarrão & 16 & 1 vez por semana \\
& Pão Francês & 28 & 2 a 4 vezes na semana \\
& Batata Inglesa & 18 & 2 a 4 vezes na semana \\
\hline \multirow{3}{*}{ Hortaliças } & Alface & 28 & 2 a 4 vezes na semana \\
& Beterraba & 15 & 2 a 4 vezes na semana \\
& Chuchu & 15 & 2 a 4 vezes na semana
\end{tabular}




\begin{tabular}{|c|c|c|c|}
\hline & Couve & 15 & 2 a 4 vezes na semana \\
\hline & Tomate & 27 & 2 a 4 vezes na semana \\
\hline \multirow{4}{*}{ Frutas } & Banana & 23 & 2 a 4 vezes na semana \\
\hline & Maçã & 23 & 2 a 4 vezes na semana \\
\hline & Laranja/Tangerin & 22 & 2 a 4 vezes na semana \\
\hline & $\mathrm{a}$ & & \\
\hline Leite e & Leite Integral & 14 & 1 vez ao dia \\
\hline Derivados & Iogurte & 17 & 2 a 4 vezes na semana \\
\hline Leguminosas & Feijão & 28 & 2 ou mais vezes ao dia \\
\hline \multirow{3}{*}{$\begin{array}{c}\text { Carne e } \\
\text { Ovos }\end{array}$} & Carne bovina & 17 & 1 vez ao dia \\
\hline & Frango & 30 & 2 a 4 vezes na semana \\
\hline & Ovo & 28 & 2 a 4 vezes na semana \\
\hline \multirow{3}{*}{$\begin{array}{l}\text { Óleos e } \\
\text { Gorduras }\end{array}$} & Óleo vegetal & 14 & 2 ou mais vezes ao dia \\
\hline & Manteiga & 20 & 2 a 4 vezes na semana \\
\hline & Azeite & 20 & 1 vez ao dia \\
\hline \multirow{4}{*}{ Outros } & Açucares & 23 & 2 ou mais vezes ao dia \\
\hline & Café & 36 & 2 ou mais vezes ao dia \\
\hline & Salgados & 25 & 1 vez na semana \\
\hline & Suco Natural & 23 & 2 a 4 vezes na semana \\
\hline
\end{tabular}

Fonte: Elaborado pelos autores, 2017.

\section{DISCUSSÃO}

Em um estudo realizado por Morgado, Rocha e Ferreira (2016) com 27 alunos do Curso de Formação de Sargentos do Exército Brasileiro relataram que apesar da exigência, aos militares, boa parte deles tinha problemas de sobrepeso e obesidade e em idades bem novas, entre 18 e 28 anos, o que foi evidenciado também no estudo de Pereira (2007) onde participaram 36 militares do $18^{\circ}$ Batalhão Logístico de Campo Grande-MS e registrou $41 \%$ dos participantes com média de 32,8 anos com sobrepeso, assim como neste estudo, onde a maior parte dos bombeiros apresentaram IMC compatível com sobrepeso ou obesidade.

Todavia deve-se pensar que o IMC não pode ser usado isoladamente para se avaliar o estado nutricional, pois o peso usado para cálculo é o peso total, que não permite diferenciar massa magra de massa gorda, o que pode interferir na classificação do estado nutricional do individuo, uma vez que, sabe-se que o tecido muscular é mais denso que o tecido adiposo (ABESO, 2016).

A pesquisa Vigitel Brasil 2014, vigilância de fatores de risco e proteção para doenças crônicas por inquérito telefônico publicado em 2015, identificou que $17 \%$ da população brasileira adulta, investigada, apresenta obesidade em algum estágio e indicou que a obesidade tem aumentado conforme a população vai atingindo idade próxima aos 44 anos.

No tocante ao percentual de gordura corpórea o estudo de Morgado, Rocha e Ferreira (2016) apontaram que $3,6 \%$ dos militares apresentaram percentual de gordura moderadamente alto, já no estudo de Damasceno et al. (2016) realizado com 25 militares do Ceará, $44 \%$ dos participantes também apresentaram um percentual de gordura classificado como moderadamente alto, no entanto os resultados encontrados pelos autores supracitados divergem dos encontrados neste estudo, onde $42,86 \%$ das 
mulheres e 21,28\% dos homens apresentaram percentual de gordura classificado como muito alto, dado relativamente pior aos encontrados pelos autores supracitados.

Em relação à CC, Tahan e Pereira (2015) realizaram um estudo onde $70 \%$ dos militares apresentaram risco de doenças cardiovasculares, já os dados da RCQ apontaram que $25 \%$ dos avaliados tinha risco para essas doenças associadas à obesidade. Esses dados corroboram com os encontrados neste estudo uma vez que a maioria dos bombeiros homens e mulheres apresentam risco moderadamente alto e alto, para o desenvolvimento de doenças cardiovasculares associadas ao sobrepeso e obesidade. O estudo de Oliveira e Marin (2003) realizado com 56 bombeiros de Maringá-PR identificou que o sobrepeso correspondia a $30,78 \%$ dos avaliados, e um risco de doenças cardiovasculares, de acordo com a CC de $11,85 \%$.

Lucena (2014) realizou um estudo com 216 militares da cidade de Campina Grande-PB onde observou que a maioria dos bombeiros avaliados apresentou alto risco cardiovascular, excesso de peso e uma grande parcela dos profissionais apresentaram hábitos não saudáveis como uso de bebida alcoólicas, cigarro ou não praticavam exercícios físicos, divergente, em partes, deste estudo em que, a maioria era praticante de atividade física regularmente, não fumavam, porém bebiam e não apresentaram risco para doenças cardiovasculares, analisando a CC isoladamente, contudo quando analisado a relação RCQ o risco para comorbidades relacionadas à obesidade foi classificado como alto para a maioria, corroborando com os resultados encontrados por Reis Neta, Fernandes Filho e Cortez (2016) em um estudo realizado com 51 militares de Floriano-PI onde foram classificados como fora da normalidade populacional quando se trata do percentual de gordura, da RCQ, do IMC e inativos segundo o Questionário Internacional de Atividade Física (IPAQ), apresentando alto ou moderado risco à saúde.

Silva, Assis e Silva (2014) realizaram um estudo com 2635 militares e identificaram que os militares tinha um perfil antropométrico mais adequado (56,39\% eutróficos) quando comparado aos estudos apresentados da população brasileira. Relataram que as exigências de capacitação física dos militares, tanto para o seu ingresso como para a sua permanência, seja, talvez, uma explicação para os resultados encontrados. Diferente desses dados os resultados encontrados neste estudo, em que as exigências aos militares são as mesmas, o perfil antropométrico indicando militares com sobrepeso e obesidade foi de $53,7 \%$.

Santos e Meireles (2015) realizaram um estudo com 20 militares do exército brasileiro e observaram que algumas inadequações dietéticas estão presentes no dia a dia dos militares, independentemente de sua aptidão física e apontaram a necessidade de maiores esclarecimentos nutricionais aos militares para corrigir inadequações dietéticas. Observações semelhantes foram realizadas neste estudo, em que existe um maior consumo de alimentos calóricos e menor consumo de alimentos ricos em fibras, pouca variedade entre os tipos de verduras e frutas, café e açúcar entre os alimentos consumidos pelos bombeiros. Os resultados ainda 
são corroborados pelo estudo realizado por Sedicías (2016) com 57 militares de Recife-PE onde observou que os hábitos alimentares e o estado nutricional dos avaliados estavam inadequados para os militares.

O estudo realizado por Lima et al. (2016) com 81 militares de Russas-CE mostram um elevado nível de sobrepeso em militares, situação preocupante, uma vez que esses fatores são relevantes para o aumento do risco de doenças cardiovasculares e metabólicas, assim como para o desenvolvimento de outros problemas de saúde, como as condições crônicas como diabetes mellitus, hipertensão, que são responsáveis por uma elevada taxa de morbimortalidade no Brasil.

\section{CONCLUSÃO}

A presença de fatores de risco para doenças cardiovasculares, sobrepeso e obesidade apesar de preocupante entre os militares, face às características da profissão, em espacial daqueles que exercem funções operacionais, é uma ocorrência em todo o Brasil, o que pode representar um risco para saúde dos profissionais e para manutenção da segurança em função de limitações físicas impostas pela presença da obesidade e sobrepeso que podem influenciar, por exemplo, na agilidade do profissional.

Com a detecção de fatores de risco cardiovasculares, limitadores de atividade física entre militares e padrões de vida não saudáveis, é importante a programação de atividades de intervenções preventivas, com enfoque nos hábitos alimentares para que os problemas relacionados a dieta inadequada e ganho de peso sejam controlados a fim de evitar que os bombeiros militares sejam impedidos de atuarem em função do agravamento de fatores de risco cardiovasculares, obesidade e surgimento de condições crônicas de saúde não transmissíveis associadas ao estilo de vida e ao padrão antropométrico dos bombeiros.

\section{REFERÊNCIAS}

ABESO. Associação Brasileira para o Estudo da Obesidade e da Síndrome Metabólica

Diretrizes brasileiras de obesidade. - 4.ed. São Paulo, SP, 2016, 188p. Disponível em: http://www.abeso.org.br/uploads/downloads/92/57fcc c403e5da.pdf. Acesso em: 15 jan. 2018.

CONFORTIN, F. G.; SOEIRO, M. Estado nutricional dos alunos Soldados Bombeiros Militares versus Soldados Bombeiros do Oeste do estado de Santa Catarina. Revista Brasileira de Nutrição

Esportiva, São Paulo, v.8, n. 44, p. 103-109, mar./abr., 2014. Disponível em:

http://www.rbne.com.br/index.php/rbne/article/view/ 440/407. Acesso em: 28 nov. 2017.

DAMASCENO, R. K. V. et al. Composição Corporal e Dados Antropométricos de Policiais Militares do Batalhão de Choque do Estado do Ceará. Saúde e Desenvolvimento Humano, v. 4, n. 2, p. 109-119, nov. 2016. Disponível em: http://dx.doi.org/10.18316/2317-8582.16.35. Acesso em: 25 nov. 2017.

DURNIN, J. V. A.; WORSLEY, J. Body fat assessed from total body density and its estimation from skinfold thickness: measurements on $481 \mathrm{men}$ and women aged from 16 to 72 years. British Journal of Nutrition. v. 32, n. 1, p. 77-97, 1974. Disponível em: https://doi.org/10.1079/BJN19740060. Acesso em: 14 dez. 2017.

JESUS, B. P. et al. Relação entre nível de atividade física, condições de saúde e ocupacionais entre bombeiros militares. Revista da Universidade Vale do Rio Verde, v. 13, n. 1, p. 77-86, 2015. Disponível em: http://dx.doi.org/10.5892/ruvrd.v13i1.1841. Acesso em: 17 dez. 2017.

KINDINGER, B. A. Condição física dos bombeiros militares do curso de formação de soldado 2013 da região metropolitana de Curitiba-PR. Trabalho de Conclusão de Curso. Graduação em Educação Física. 
Universidade Tecnológica Federal do Paraná. Curitiba, PR, 2015, 44p.

LIMA, A. D. et al. A associação do índice de massa corpórea com a relação cintura/quadril no comportamento da saúde de policiais militares do Estado do Ceará. Revista Brasileira de Prescrição e Fisiologia do Exercício, São Paulo, v. 10, n. 59, p. 330-339, maio/jun., 2016. Disponível em: http://www.rbpfex.com.br/index.php/rbpfex/article/vi ew/944/795. Acesso em: 17 dez. 2017.

\section{LUCENA, M. M. Risco Cardiovascular em} Policiais e Bombeiros de Campina Grande Paraíba. Trabalho de Conclusão de Curso. Graduação em Farmácia. UEPB, Campina Grande, PB, 2014, 35p.

MORGADO, J. J. M.; ROCHA, M. F. F.; FERREIRA, M. E. C. Efeitos do Treinamento Físico Militar nas características antropométricas e no desempenho físico de militares. Rev Ed Física / J Phys Ed, v. 85, n. 4, p. 376-386, 2016. Disponível em:

http://177.38.96.106/index.php/revista/article/view/1 91/pdf 61. Acesso em: 15 jan. 2018.

OLIVEIRA, A. A. B.; MARIN, I. M. C. Avaliação do estado nutricional da corporação de bombeiros de Maringá e implantação de um programa de educação nutricional. Iniciação Científica Cesumar, v. 5, n. 2, p. 95-102, jul./dez. 2003. Disponível em: http://periodicos.unicesumar.edu.br/index.php/iccesu mar/article/view/72/44. Acesso em: 13 nov. 2017.

\section{PEREIRA, M. M. Análise da aptidão} cardiorrespiratória, composição corporal e da capacidade de trabalho dos oficiais, subtenentes e sargentos do $18^{\circ}$ batalhão logístico. Universidade Federal de Mato Grosso do Sul. Campo Grande, MS, 2007.

POLLOCK, M. L; WILMORE, J. H. Exercícios na saúde e na doença. Avaliação e prescrição para prevenção e reabilitação. $2^{\mathrm{a}}$ ed. Rio de Janeiro: MEDSI, 1993.

REIS NETA, E. S. D. A.; FERNANDES FILHO, J.; CORTEZ, A. C. L. Nível de atividade física e estado nutricional de policiais militares na cidade de Floriano-PI. Revista Kinesis, v. 34, n. 1, p. 84-101, jan./jun. 2016. Disponível em: http://dx.doi.org/10.5902/2316546422273. Acesso em: 10 jan. 2018.

SANTOS NETO, T.; CAVALCANTE NETO, J. L.; PINTO, M. P. Estresse e níveis de atividade física de Bombeiros militares de Alagoas, Brasil. R. bras. Qual. Vida, Ponta Grossa, v. 6, n. 4, p. 250-260, out./dez., 2014. Disponível em:
http://dx.doi.org/10.3895/S2175-

08582014000400006. Acesso em: 20 dez. 2017.

SANTOS, D.; MEIRELLES, C. Avaliação do perfil dietético dos militares do Centro de Capacitação Física do Exército. Rev Ed Física / J Phys Ed, v. 84, n. 1, p. 25, 2015. Disponível em: http://177.38.96.106/index.php/revista/article/view/1 06/78. Acesso em: $11 \mathrm{dez} .2017$.

\section{SEDICÍAS, L. M. D. S. Hábitos alimentares e} estado nutricional dos policiais militares da cavalaria da região metropolitana do município de Recife, Pernambuco. Trabalho de Conclusão de Curso. Graduação em Nutrição. Universidade Federal de Pernambuco. Vitória de Santo Antão, PE, 2016, 42p.

SILVA, M. E. N.; ASSIS, J. N.; SILVA, J. R. Perfil nutricional dos militares de uma unidade militar da cidade de Anápolis em Goiás. Revista Brasileira de Nutrição Esportiva, São Paulo, v. 8, n. 48, p. 354362, nov./dez., 2014. Disponível em: http://www.rbne.com.br/index.php/rbne/article/view/ 478/439. Acesso em: 27 nov. 2017.

TAHAN, F.; PEREIRA, J. C. Avaliação de risco cardiovascular por indicadores antropométricos em policiais militares de um batalhão do Sul de Minas Gerais. Nutrição Brasil, v.14, n. 4, 2015.

VIGITEL BRASIL 2014. Vigilância de fatores de risco e proteção para doenças crônicas por inquérito telefônico. Secretaria de Vigilância em Saúde, Departamento de Vigilância de Doenças e Agravos não Transmissíveis e Promoção da Saúde. - Brasília, DF : Ministério da Saúde, 2015, 154p. Disponível em:

http://bvsms.saude.gov.br/bvs/publicacoes/vigitel_bra sil_2014.pdf. Acesso em: 18 jan. 2018.

WHO. World Health Organization. Obesity. Preventing and Managing the Global Epidemic. Report of a WHO Consultation on Obesity, Geneva: World Health Organization; 1999. p. 107-158.

Rejane Mendes Costa Borges
Graduada em Nutrição. Faculdades de Saúde
Ibituruna, FASI.

Maria Cecília Andrade Silva

Graduada em Nutrição. Faculdade de Saúde Ibituruna, FASI.

\begin{tabular}{l}
\hline Simone Valéria Dias Souto \\
Educadora Física Doutora em Ciências do Desporto. \\
\hline
\end{tabular}


Ernandes Gonçalves Dias

Enfermeiro Mestre em Ciências. Especialista em Enfermagem do Trabalho e Docência na Saúde. 